\title{
Imaging in urinary tract infection: top-down or down-up?
}

\author{
Lil-Sofie Ording Müller
}

(C) The Author(s) 2011. This article is published with open access at Springerlink.com

\section{Introduction}

Paediatric uroradiology is one of the mainstays of daily radiological practice with strong traditions in work-up for urinary tract infection (UTI). For decades, children with UTI were almost inevitably imaged from top down with intravenous urography and renal technetium-99 m dimercaptosuccinic acid (DMSA) scintigraphy to diagnose and localise UTI in the acute phase, and subsequently from down up with voiding cystourethrography (VCUG) to look for vesicoureteral reflux (VUR). Accurate clinical tests have now reduced the role of imaging for primary diagnosis in UTI. Moreover, the view on VUR has changed over the last decade. Previously, VUR was perceived as a homogenous condition that posed an equal risk of renal damage in all children. We now know that low-grade VUR is not necessarily associated with renal damage in patients with UTI, hence routine imaging to assess VUR is no longer recommended for all children with UTI. The outcome of higher-grade VUR appears also to depend on individual characteristics of the patients, and not only on the grade of VUR per se. Renal scarring can even occur in patients without demonstrable VUR. The focus for imaging tests has therefore shifted from a down-up towards a top-down approach, focusing more on the impact of UTI on the kidneys and less on assessment for VUR. The question is not only whether to image top-down or down-up, but also who will need imaging tests and when should the tests be performed?

Disclaimer Dr. Müller has no financial interests, investigational or off-label uses to disclose.

L.-S. O. Müller $(\bowtie)$

University Hospital North Norway and Great Ormond Street

Hospital for Children,

Great Ormond Street,

London WC1N 3JH, UK

e-mail: lil-sofie.ording.muller@unn.no

\section{Objective of imaging in UTI}

The aim of the primary investigations should be to detect underlying conditions that may render the patient more susceptible to renal damage caused by the infection, e.g., signs of atypical UTI, and urinary tract malformations. The follow-up scans should assess renal damage caused by infection, e.g., scarring, abnormal parenchymal echogenicity, cyst formation and renal growth impairment. The modalities routinely used, US, VCUG and DMSA, are today applied more selectively with more individualised indications to take into consideration the known risk factors for renal damage. This is reflected both in the United Kingdom (National Institute of Clinical Excellence [NICE]) and in the United States (American College of Radiologists) guidelines for imaging in childhood UTI. Both guidelines differentiate between neonates and older children, and recommend specific imaging algorithms in children with risk factors for renal damage, such as atypical UTI, family history of VUR, poor urine flow or hydronephrosis on US. Similar imaging recommendations have also been made by the European Society for Uroradiology/European Society of Paediatric Radiology-working party (Fig. 1). Investigations should be economically justifiable, reliable and as noninvasive as possible. Computed tomography and magnetic resonance imaging currently have no place in routine work-up of UTI in childhood.

\section{Imaging for diagnosing and grading UTI}

NICE guidelines do not recommend imaging to differentiate upper and lower UTI. In younger patients, though, this differentiation is difficult based on clinical and laboratory findings alone. DMSA has traditionally been the modality of choice to assess renal involvement in UTI. 


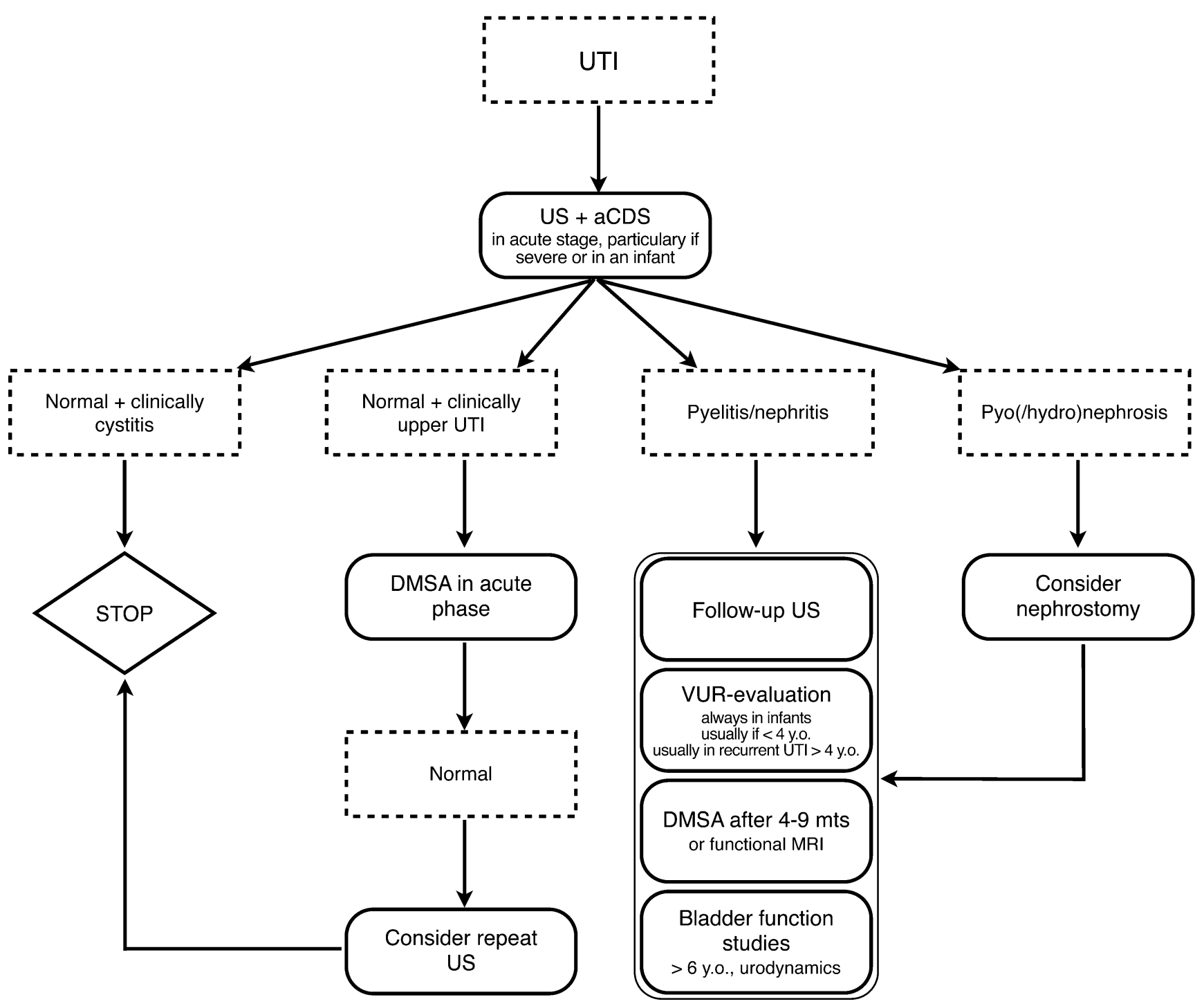

Fig. 1 Modified imaging algorithm in children with urinary tract infection (UTI), based on the algorithm made by the ESPR/ESUR taskforce group, published in 2007. A reliable clinical/laboratory diagnosis of UTI is an essential entry criterion for imaging, and should be based on leucocyturia and nitrite positive urine; positive cultures $(>104$ from catheter sample, $>106$ from voiding); leucocytosis in blood and elevated

C-reactive protein in serum. Equivocal findings or suspicion of complicated stone disease, complicated UTI (e.g., xantogranulomatous pyelonephritis, tuberculosis, abscess) or tumour indicate further evaluation with computed tomography or magnetic resonance imaging. US Ultra- sound, $a C D S$ amplitude-coded colour Doppler sonography, DMSA static renal scintigraphy, VUR vesicoureteral reflux, $m t s$ months, y.o. years old

However, modern US with added amplitude-coded colour Doppler sonography (aCDS) has high sensitivity for the diagnosis of renal involvement in UTI, and should be the first modality of choice when imaging in the acute phase (Fig. 2). Renal MR will possibly gain momentum in the future in diagnosing and grading.

\section{Renal technetium-99 m DMSA scintigraphy}

DMSA can give false-negative results for acute renal involvement, particularly in poorly functioning or hydronephrotic kidneys, scarred kidneys, in the hyper acute phase,

or with small lesions in older patients. As advances in modern US are implemented in clinical practice, the use of DMSA in acute infection will potentially be limited to selected cases. The main role of DMSA in paediatric UTI imaging is to look for renal damage caused by infection, and it should be performed 4-6 months after treatment (Fig. 3).

\section{Ultrasound}

US is the workhorse of paediatric genitourinary imaging and is always the first (and sometimes the only) modality needed. It should be performed by an investigator experienced in 


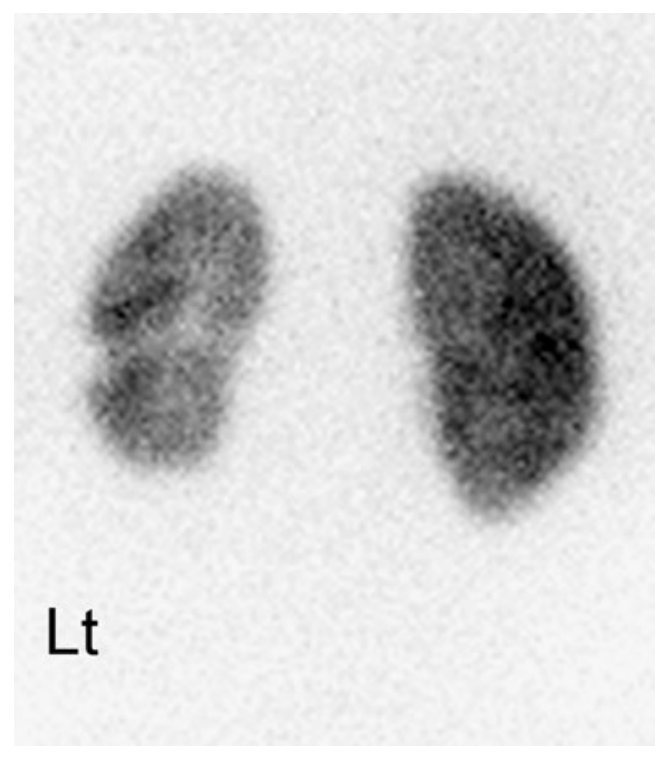

Fig. 2 Static renal technetium- $99 \mathrm{~m}$ dimercaptosuccinic acid (DMSA) scintigraphy 6 months after an upper urinary tract infection in a 6-year-old girl shows a focal, photopenic defect in the lower pole of the left kidney, suggestive of renal scarring. Courtesy of Dr. Marina Easty, Great Ormond Street Hospital, UK

paediatric US. The child should be well hydrated for the US, and pre- and post-void assessment must be included.

\section{Voiding cystourethrography}

The indications for VCUG have changed with new knowledge of the relevance of VUR. VUR assessment is most important in younger age groups, particularly in infants. VCUG is less frequently indicated in older children, but should be performed when urethral pathology or a complex urinary tract malformation is suspected. VUR assessment is also important in children with significant hydronephrosis and in recurrent or atypical UTI. Pulsed fluoroscopy with last-image hold is recommended to reduce the radiation burden. If available, contrast-enhanced voiding urosonography can replace VCUG as follow-up examination, for first assessment of VUR in girls and for (family) screening.

\section{Conclusion}

Radiological imaging in UTI remains crucial to detect underlying pathology and to monitor the kidneys to prevent renal damage and scarring. Importantly, patients, particularly older children, must be meticulously selected for more invasive imaging. Whenever imaging is indicated and performed, recommendations should be followed to minimise radiation exposure and to provide optimal management of children with UTI at reasonable cost.

Open Access This article is distributed under the terms of the Creative Commons Attribution Noncommercial License which permits any noncommercial use, distribution and reproduction in any medium, provided the original author(s) and source are credited.
Fig. 3 Gray-scale ultrasound (a) of acute pyelonephritis, showing an enlarged, swollen kidney, with distorted echostructure and reduced corticomedullary differentiation. Amplitude-coded colour Doppler sonography (aCDS) (b) of the same kidney demonstrates perfusion defects in the affected area (arrowheads) during the acute pyelonephritis. Courtesy of Dr. Michael Riccabona, University Hospital Graz, Austria
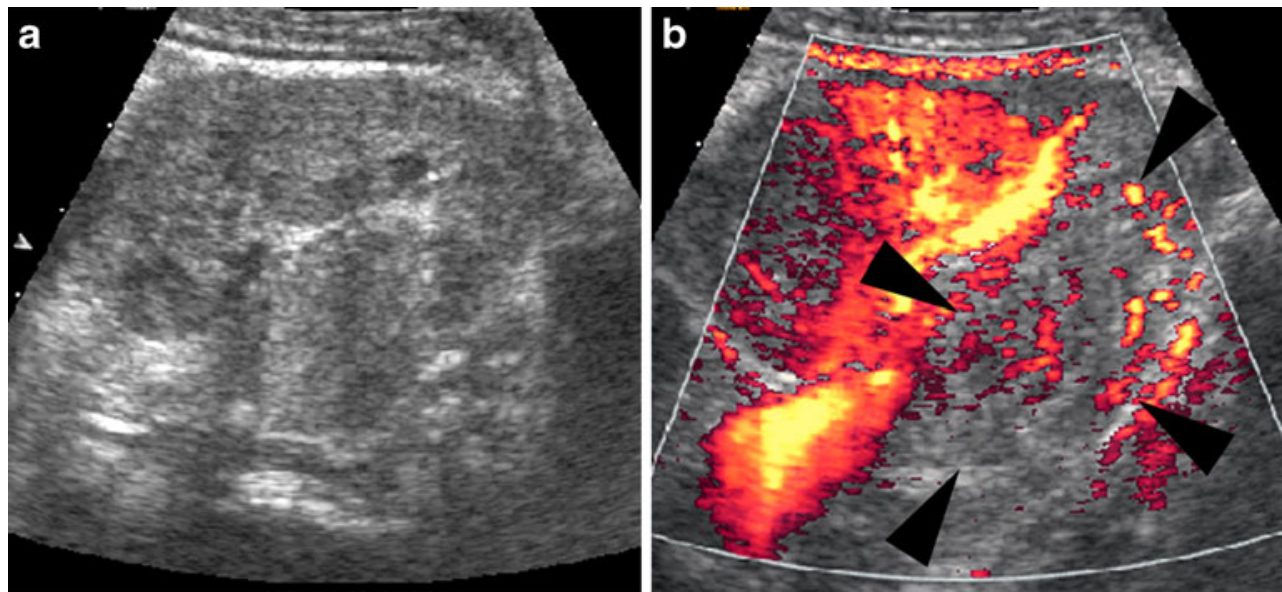